\title{
Circ_RPL23A acts as a miR-1233 sponge to suppress the progression of clear cell renal cell carcinoma by promoting ACAT2
}

\section{Liang Cheng}

The First Affiliated Hospital of Jiamusi University

\section{Huifeng Cao}

The First Affiliated Hospital of Jiamusi University

Jianbo Xu

The First Affiliated Hospital of Jiamusi University

\section{Mo Xu}

The First Affiliated Hospital of Jiamusi University

\section{Wenjie He}

The First Affiliated Hospital of Jiamusi University

\section{Wenjing Zhang}

The First Affiliated Hospital of Jiamusi University

\section{Longxin Dong}

Jiamusi University

\section{Dayin Chen ( $\nabla$ svb5mlr@163.com )}

The First Affiliated Hospital of Jiamusi University

\section{Research}

Keywords: Circ_RPL23A, clear cell renal cell carcinoma, miR-1233, ACAT2

Posted Date: March 20th, 2020

DOI: https://doi.org/10.21203/rs.3.rs-18104/v1

License: (c) (1) This work is licensed under a Creative Commons Attribution 4.0 International License. Read Full License

Version of Record: A version of this preprint was published at Journal of Bioenergetics and Biomembranes on May 25th, 2021. See the published version at https://doi.org/10.1007/s10863-02109901-8. 


\section{Abstract}

Background Clear cell renal cell carcinoma (CCRCC) is a prevalent urological carcinoma with high metastatic risk. Circular RNAs (circRNAs) have been identified as effective diagnostic and therapeutic biomarkers for CCRCC. This research aims to disclose the involvement of circRNA ribosomal protein L23a (circ_RPL23A) in CCRCC, and how it regulates carcinogenesis. Methods We performed quantitative realtime polymerase chain reaction (qRT-PCR) to examine circ_RPL23A, microRNA-1233 (miR-1233) and acetyl-coenzyme A acetyltransferase 2 (ACAT2). Cellular behavior detection included cell cycle, proliferation, apoptosis and metastasis, which were severally analyzed using propidium iodide (PI)-flow cytometry, 3-(4, 5-dimethylthiazol-2-y1)-2, 5-diphenyl tetrazolium bromide (MTT), Annexin V/PI-flow cytometry and transwell migration/invasion assays. ACAT2 and related proteins of cell cycle, epithelialmesenchymal transition (EMT) were measured via western blot. Target relationship was analyzed via dual-luciferase reporter assay. A xenograft model was used to study circ_RPL23A action in mice. Results Both in CCRCC tissues and cells, circ_RPL23A had a down-regulated tendency. Explicitly, circ_RPL23A overexpression inhibited cell cycle, proliferation and metastasis but enhanced apoptosis of CCRCC cells, whereas these effects of circ_RPL23A were dependent on the suppression of its target miR-1233. Besides, miR-1233 could target ACAT2 and circ_RPL23A regulated ACAT2 by sponging miR-1233. Also importantly, miR-1233 was a pro-cancer gene in CCRCC via targeting ACAT2. CCRCC tumor growth was also decreased in vivo by circ_RPL23A through miR-1233/ACAT2 axis. Conclusion Altogether, the circ_RPL23A/miR-1233/ACAT2 axis in this report provides an in-depth cognition for CCRCC pathogenesis and circ_RPL23A may has pivotal value in diagnosing and treating CCRCC.

\section{Introduction}

Renal cell carcinoma (RCC), a large class of cancers originated from renal tubular epithelial cells, is ranked as the third most common malignant neoplasm in genitourinary system [1]. It is categorized into various subtypes, mainly including clear cell RCC (CCRCC, 75\%), papillary RCC (15\%), chromophobe RCC $(5 \%)$ and other types less than $1 \%[2,3]$. As the most familiar RCC subtype, the incidence and mortality of CCRCC have rapidly increased in clinical presentation on account of its high metastasis peculiarity [4]. And the distant metastasis and local relapse cause the low overall survival and dissatisfactory prognostic effect on CCRCC patients [5]. In the last decades, molecular targeted therapy has been applied in treating a myriad of cancers [6, 7]. Hence, to identify novel susceptive molecules appears to be an evangel for CCRCC treatment.

Circular RNAs (circRNAs) are covalent closed non-coding RNAs (ncRNAs) without 5' cap and 3 ' tail in eukaryotes [8]. Dysregulated circRNAs have been recognized to participate in the oncogenesis and malignance of different kinds of human cancers $[9,10]$. CircRNA ribosomal protein L23a (circ_RPL23A, has_circ_0092360), a newly discovered circRNA, was aberrantly down-regulated in clinical CCRCC samples [11]. Nevertheless, its biological function is still unknown. 
CircRNAs usually serve as "miRNA sponges" to mediate cancer progression [12]. MiRNAs are another genre of ncRNAs involved in the cellular processes of cancers and its regulation is achieved by the combination with the $3^{\prime}$-untranslated regions (3'-UTRs) of target mRNAs, thus affecting corresponding gene expression [13]. Interestingly, the circRNA/miRNA/mRNA functional axis has been found in cancer process, such as osteosarcoma [14], hepatocellular carcinoma [15], papillary thyroid carcinoma [16], and CCRCC [11]. MiR-1233 was reported to be overexpressed $[17,18]$ and ACAT2 was a down-regulated gene [19] in CCRCC. We assume there is a similar network in circ_RPL23A, miR-1233 and ACAT2 in CCRCC, and this study will be performed to verify it.

\section{Materials And Methods}

\section{Tissue collection and ethical statement}

CCRCC tissues were acquired from clinical patients diagnosed with CCRCC in the period of their surgical resection at First Affiliated Hospital of Jiamusi University, then preserved in liquid nitrogen right away. Before this study was started, we have collected 60 CCRCC samples and an equal number of normal para-carcinoma tissues.

All individuals were entirely informed regarding the objective of this research. Tissue collection was performed after the informed consent was signed and in line with the Declaration of Helsinki. Besides, our study has got the authorization of the Ethical Committee of First Affiliated Hospital of Jiamusi University.

\section{Cell culture and transfection}

For cell culture, human renal tubular epithelial cell line HK-2 and CCRCC cell lines (786-0, 769-P, CAKI-1 and A498) purchased from American Type Culture Collection (ATCC, Manassas, VA, USA) were cultured using Dulbecco's modified eagle medium (DMEM; Hyclone, Logan, UT, USA) complemented with $10 \%$ fetal bovine serum (FBS; Corning Inc., Corning, NY, USA) and 1\% penicillin/streptomycin (Millipore, Billerica, MA, USA) in a humidified incubator. The growing environment was set as the temperature at $37^{\circ} \mathrm{C}$ and $\mathrm{CO}_{2}$ of $5 \%$.

For cell transfection, the mixtures of Lipofectamine3000 reagent (Invitrogen, Carlsbad, CA, USA) and vectors or oligonucleotides were pipetted into 786-0 and 769-P cells in 96-well plates as per the user's guideline. The circ_RPL23A overexpression vector (circ_RPL23A), miR-1233 mimic/inhibitor (miR1233/anti-miR-1233), small interfering RNA (siRNA) targeting ACAT2 (si-ACAT2) and the respective negative controls (Vector, miR-NC, anti-miR-NC and si-NC) were all obtained from RIBOBIO (Guangzhou, China).

\section{Quantitative real-time polymerase chain reaction (qRT-PCR)}

Trizol (Sangon, Shanghai, China) was employed for the isolation of total RNA from collected tissues and cells, and the reverse transcription assay ( $1 \mu \mathrm{g}$ RNA/sample) was instantly implemented using 
QuantiTect Reverse Transcription Kit (Qiagen, Hilden, Germany). Then the qRT-PCR was carried out by QuantiFast SYBR ${ }^{\circledR}$ Green PCR Kit (Qiagen) via the QuantStudio ${ }^{\text {TM }} 6$ Flex Real-Time PCR System (96-well; Applied Biosystems, Carlsbad, CA, USA). And the cycle threshold method $\left(2^{-\Delta \Delta C t}\right)$ was exploited to analyze the relative expression levels of related molecules. Glyceraldehyde-3-phosphate dehydrogenase (GAPDH) acted as the internal control of circRNA and mRNA, while U6 was used for miRNA normalization. The used primers (Sangon) included circ_RPL23A: Forward (F), 5'AAGGAACCACTGATGCACCT-3' and Reverse (R), 5'-TTCCCAATTCGTTCGCTCT-3'; RPL23A: F, 5'CCCTTTCGTCACAAGATGGCG-3' and R, 5'-GAATTAGGCAGCTGGACTCA-3'; miR-1233: F, 5'TGGGAGGCCAGGGCAC-3'; ACAT2: F, 5'-CATGCTGCTGCTCATCTTCT-3' and R, 5'ACTGCGGAGACCAGGAACA-3'; GAPDH: F, 5'-ACCCACTCCTCCACCTTTG-3' and R, 5'CTGTAGCCAAATTCGTTGTCAT-3'; U6: F, 5'- CTCGCTTCGGCAGCACA-3' and R, 5'AACGCTTCACGAATTTGCGT-3'.

\section{Stability analysis}

Actinomycin D (Millipore), a transcription inhibitor, was added to the culture medium for 786-0 and 769-P cells with a concentration of $2 \mathrm{mg} / \mathrm{mL}$ for $0 \mathrm{~h}, 8 \mathrm{~h}, 16 \mathrm{~h}$ and $24 \mathrm{~h}$. In other hand, $4 \mu \mathrm{g}$ total RNA was subjected to $12 \mathrm{U}$ Ribonuclease R (RNase R; Epicentre Technologies, Madison, WI, USA) digestion at $37^{\circ} \mathrm{C}$ for $1 \mathrm{~h}$. Then circ_RPL23A and RPL23A were determined via qRT-PCR post-treatment.

\section{Cell cycle detection}

Propidium iodide (PI) staining was applied for measuring the changes of cell cycle (G0/G1, S and M) through a flow cytometer (BD Biosciences, San Diego, CA, USA) as previously described [20, 21].

\section{Western blot}

The standard procedures of western blot contained: protein extraction and quantification by radioimmunoprecipitation assay (RIPA) buffer (Abcam, Cambridge, UK) and a BCA Protein Assay Kit (Abcam); protein separation via the sodium dodecyl sulfate-polyacrylamide gel electrophoresis (SDS-PAGE) for $2 \mathrm{~h}$; protein transferring to polyvinylidene fluoride (PVDF) membranes (Abcam) and non-specific binding prevention in Blocking Buffer (Abcam); antibody incubation of primary antibodies (overnight at $4{ }^{\circ} \mathrm{C}$ ) and second antibody (at room temperature for $1 \mathrm{~h}$ ); protein appearance using a ECL Substrate Kit (Abcam) and analysis by the ImageLab software version 4.1 (Bio-Rad, Hercules, CA, USA). All antibodies were purchased from Abcam and shown as follows: anti-CyclinD1 (ab16663, 1:1000), anti-Cell Cycle Dependent Kinase 4 (anti-CDK4; ab137675, 1:1000), anti-snail (ab180714, 1:1000), anti-E-cadherin (ab15148, 1:1000), anti-ACAT2 (ab131215, 1:1000), anti-GAPDH (ab9485, 1:3000) and goat anti-rabbit IgG H\&L (HRP) secondary antibody (ab205718, 1:5000). GAPDH served as an endogenous control for other proteins.

\section{3-(4, 5-dimethylthiazol-2-y1)-2, 5-diphenyl tetrazolium bromide (MTT) assay}


MTT assay was administrated for the assessment of cell proliferation. In 96-well plates, inoculated cells (about $1 \times 10^{4}$ ) for $24 \mathrm{~h}$ were transfected with different experimental groups. After $0 \mathrm{~d}, 1 \mathrm{~d}, 2 \mathrm{~d}$ and $3 \mathrm{~d}$, each well was added with $50 \mu \mathrm{L} 1 \times \mathrm{MTT}$ (KeyGen, Nanjing, China) to incubate at $37^{\circ} \mathrm{C}$ for $4 \mathrm{~h}$. Then supernatant was sucked out using a micropipette and the optical density (OD) of each well was determined at $490 \mathrm{~nm}$ through a microplate reader after the addition of $150 \mu \mathrm{L}$ dimethylsulfoxide (DMSO; Sangon).

\section{Apoptosis detection}

The detection of cell apoptosis was performed using FITC Annexin V Apoptosis Detection Kit I (BD Biosciences) through the flow cytometer (BD Biosciences). $5 \mu \mathrm{L}$ FITC Annexin V and $5 \mu \mathrm{L}$ PI were severally pipetted to $100 \mu \mathrm{L}$ cell suspension in $1 \times$ Bing Buffer $\left(1 \times 10^{5}\right.$ cells $)$. After gentle mixing and lucifugal incubation for $15 \mathrm{~min}$, apoptotic cells were discerned as cells of Annexin $\mathrm{V}+/ \mathrm{PI}$ - and Annexin $\mathrm{V}+/ \mathrm{PI}+$ in staining, followed by the calculation of apoptosis rate (the apoptotic cells/total cells $\times 100 \%$ ).

\section{Transwell assay}

The migrated and invaded cells were determined via the 24-well transwell chamber (Corning Inc.), in which cell suspension (produced by serum-free medium) in the upper chamber could be attracted to the lower chamber by 10\% FBS+DMEM medium. $24 \mathrm{~h}$ later, cell fastening and dyeing were respectively implemented by methanol and crystal violet (Sangon). Whereafter, the migrated and invaded cells were observed and photographed via an inverted microscope (Olympus, Tokyo, Japan). It should be noted that the matrigel (Corning Inc.) needed to envelop the upper chamber in invasion assay before seeding cells, while it was not necessary for migration assay.

\section{Dual-luciferase reporter assay}

The gene combination was affirmed via the dual-luciferase reporter assay. For circ_RPL23A and miR1233, wild-type (wt) or mutant-type (mut) circ_RPL23A sequence that contained the binding sites of miR1233 or mutated sites was cloned into the pGL3 luciferase basic vector (Promega, Madison, WI, USA) and named as circ_RPL23A-wt and circ_RPL23A-mut. Then they were respectively transfected with miR-1233 or miR-NC into 786-0 and 769-P cells, followed by the measurement of the dual-luciferase assay system (Promega) according to the manufacture's instruction. For ACTA2 and miR-1233, ACAT2-wt and ACAT2mut plasmids were also constructed as above, and the luciferase activity was assayed. The renilla luciferase intensity was used as the internal reference of firefly luciferase intensity.

\section{Xenograft tumor assay}

Six-week-old BALB/c nude mice $(n=10)$ were bought from Animal Center of Chinese Academy of Medical Sciences (Beijing, China) and subcutaneously injected with $2 \times 10^{6} 786-0$ cells transfected with circ_RPL23A using Vector as a negative control. After injection for $10 \mathrm{~d}$, tumor volume (length $\times$ width $^{2} \times$ $0.5)$ was measured per 5 days. Tumors were weighed at $30 \mathrm{~d}$ after mice were euthanatized by cervical 
dislocation, then qRT-PCR and western blot were adopted for examining the levels of circ_RPL23A, miR1233 and ACAT2. All animals were cared in compliance with the National Institutes of Health (USA) guidelines, and this experiment was carried out after the approval of the Institutional and Local Animal Ethics Committee of First Affiliated Hospital of Jiamusi University.

\section{Statistical analysis}

SPSS 22.0 was exploited for statistical analysis in our research. All assays were administrated in three duplications and data were shown as mean \pm standard deviation (SD). Spearman's correlation coefficient was used to analyze the linear relation between molecules. Difference analysis was executed using Student's $t$-test and one-way analysis of variance (ANOVA) followed by Tukey's test. $P$ value $<0.05$ was deemed as the indication of a significant difference.

\section{Results}

\section{Circ_RPL23A down-regulation in CCRCC was confirmed}

The gene expression microarray GSE100186 was searched from Gene Expression Omnibus (GEO) datasets and analyzed using GEO2R, a powerful tool to screen the differentially expressed genes (DEGs) between CCRCC and normal samples. As the exhibition of Fig. 1A, circ_RPL23A was a maximally downregulated gene. Then qRT-PCR was carried out to assay the expression pattern of circ_RPL23A in clinical CCRCC samples and in cellular level. The results clearly displayed the low expression of circ_RPL23A in CCRCC tissues (Fig. 1B) and cells (786-0, 769-P, CAKI-1 and A498) (Fig. 1C), using non-carcinoma tissues and HK-2 cell line as respective negative control. 786-O and 769-P cells expressed with lower circ_RPL23A among four CCRCC cell lines were chosen for following research. After transcription inhibition by Actinomycin D, the half-life of circ_RPL23A (more than $24 \mathrm{~h}$ ) was found to be longer than that of RPL23A (about 16 h) (Fig. 1D-E). And different from RPL23A, circ_RPL23A has great resistance to RNase R digestion (Fig. 1F-G). The high stability provided the evidence to verify circ_RPL23A as a circRNA.

\section{Overexpression of circ_RPL23A repressed tumor progression in CCRCC cells}

The circ_RPL23A overexpression vector was constructed for studying what role circ_RPL23A played in CCRCC, and qRT-PCR analysis showed the 7-fold increased changes of circ_RPL23A transfection in 786-0 and 769-P cells (Fig. 2A). As a result of circ_RPL23A overexpression, cell number in G0/G1 was augmented while that in $S$ phase was reduced according to cell cycle detection (Fig. 2B-C). This circ_RPL23A-induced cell cycle arrest was further validated by western blot, in which cell cycle proteins CyclinD1 and CDK4 were significantly inhibited after up-regulation of circ_RPL23A (Fig. 2D-E). By implementing MTT and Annexin V-PI flow cytometry, we observed the lower cell proliferation ability (Fig. $2 \mathrm{~F}-\mathrm{G}$ ) and higher apoptotic rate (Fig. 2H) in circ_RPL23A group than these in Vector group. As for the influence of circ_RPL23A on cell metastasis, migrated (Fig. 2I) and invaded (Fig. 2J) cells were found to be markedly decreased in transwell assay. Moreover, the down-regulated snail (an epithelialmesenchymal transition (EMT)-promoting marker) and up-regulated E-cadherin (an EMT-inhibitory 
marker) in 786-0 and 769-P cells transfected with circ_RPL23A also demonstrated the metastasis inhibition by overexpressed circ_RPL23A (Fig. 2K-L). Collectively, circ_RPL23A played a suppressive role in CCRCC progression.

\section{Circ_RPL23A was identified as a miR-1233 sponge in CCRCC}

In circinteractome, we noticed seven binding sites between circ_RPL23A (AGGGCUC) and miR-1233 (UCCCGAG) (Fig. 3A). After the sites in circ_RPL23A were mutated into CUUUAGA, luciferase reporter plasmids circ_RPL23A-wt and circ_RPL23A-mut were generated for administrating the dual-luciferase reporter assay. As the illustration of Fig. 3B-C, the luciferase signal of circ_RPL23A-wt+miR-1233 group was considerably lower than that of circ_RPL23A-wt+miR-NC group, whereas there was no conspicuous change tendency between circ_RPL23A-mut+miR-1233 and circ_RPL23A-mut+miR-NC groups. And transfection of circ_RPL23A led to the inhibition of miR-1233 level, suggesting that circ_RPL23A could directly sequester miR-1233 (Fig. 3D). About the expression of miR-1233 in CCRCC tissues, we found its up-regulated trend that was opposite from circ_RPL23A (Fig. 3E), and the linear relationship between them was negative $(r=-0.6939, P<0.001)$ (Fig. 3F). 786-0 and 769-P cells also expressed the high-level miR-1233 contrasted to HK-2 cells (Fig. 3G). Taken together, miR-1233 could be sponged by circ_RPL23A.

\section{Circ_RPL23A/miR-1233 inhibited the development of CCRCC}

After circ_RPL23A was identified as a sponge of miR-1233 in CCRCC cells, we further analyzed whether miR-1233 was associated with the inhibitory effect of circ_RPL23A on CCRCC. In 786-0 and 769-P cells, circ_RPL23A-induced miR-1233 inhibition was abrogated by miR-1233 mimic transfection, which indicated that the transfection efficiency of miR-1233 was excellent (Fig. 4A). After the addition of miR1233 in circ_RPL23A-overexpressed cells, original cell cycle arrest (Fig. 4B-C) and the down-regulated cell cycle proteins (CyclinD1 and CDK4) (Fig. 4D-E) were evidently assuaged. Also, the proliferation inhibition (Fig. 4F-G) and apoptosis promotion (Fig. 4H) in 786-0 and 769-P cells transfected with circ_RPL23A were ameliorated following the introduction of miR-1233 mimic. And, circ_RPL23A sponged miR-1233 to inhibit CCRCC cell migration (Fig. 4I), invasion (Fig. 4J) and EMT process (Fig. 4K-J). These data expounded that circ_RPL23A/miR-1233 axis restrained the tumor formation in CCRCC.

\section{Circ_RPL23A enhanced ACAT2 via sponging miR-1233 in CCRCC}

Interestingly, miRDB online software predicted the combination of miR-1233 and ACAT2 in molecular sequence (Fig. 5A) and dual-luciferase reporter assay verified their actual binding (Fig. 5B-C).

Subsequently, the effect of miR-1233 on ACAT2 was explored using miR-1233 or anti-miR-1233 transfection to overexpress or inhibit miR-1233 level (Fig. 5D). As Fig. 5E-F depicted, ACAT2 mRNA and protein levels were repressed after miR-1233 overexpression while miR-1233 inhibition triggered the promotion of them. The qRT-PCR and western blot assays suggested that ACAT2 expression was distinctly lessened in CCRCC cells (Fig. 5G-H). It was similar that ACAT2 mRNA level was down-regulated in CCRCC tissue samples (Fig. $5 \mathrm{I})$, in which there was a negative relation $(r=-0.7151, P<0.001)$ between ACAT2 mRNA and miR-1233 expression (Fig. 5J). Also, CCRCC tissues manifested the decreased ACAT2 
protein level by contrast to normal tissues (Fig. 5K). Additionally, circ_RPL23A transfection exerted the accelerative influence on ACAT2 mRNA and protein expression, while this promotion was neutralized via miR-1233 up-regulation (Fig. 5L-M). Therefore, miR-1233 could target ACAT2 and circ_RPL23A promoted the expression of ACAT2 by sequestering miR-1233.

\section{MiR-1233 played a tumor-promoting role in via targeting ACAT2 in CCRCC}

The role of miR-1233 and its functional mechanism were analyzed using rescue experiment by transfection of anti-miR-1233, anti-miR-1233+si-ACAT2 or their matched controls in 786-0 and 769-P cells. As shown in Fig. 6A-B, si-ACAT2 transfection offset the anti-miR-1233-induced up-regulation of ACAT2 mRNA and protein expression, hinting that the transfection efficiency of si-ACAT2 was in success and miR-1233 indeed could negatively regulated ACAT2. Then we found miR-1233 inhibitor hampered the transition of cells from G0/G1 to $S$ phase, but this phenomenon was mitigated after ACAT2 was knocked down (Fig. 6C-D). And anti-miR-1233-mediated CyclinD1 and CDK4 inhibition was also attenuated by siACAT2 transfection (Fig. 6E-F). Also, MTT and Annexin V-PI flow cytometry revealed that the absorbance of anti-miR-1233+si-ACAT2 group was higher (Fig. 6G-H) while the apoptotic rate was lower (Fig. 6I) than those of alone anti-miR-1233 transfection. In addition, miR-1233 down-regulation had inhibitory impacts on cell migration (Fig. 6J), invasion (Fig. 6K) and EMT (snail inhibition and E-cadherin promotion in protein expression) (Fig. 6L-M), whereas these effects were all restored after the knockdown of ACAT2. Altogether, anti-miR-1233 retarded CCRCC progression by up-regulating ACAT2, which indicated the protumor function of miR-1233 in CCRCC via targeting ACAT2.

\section{Circ_RPL23A worked as a tumor inhibitor of CCRCC in vivo by ACAT2 up-regulation as a miR-1233 sponge}

A xenograft model was established for investigating the action of circ_RPL23A in vivo. In comparison to control group, the tumor volume (Fig. 7A) and weight (Fig. 7B) of circ_RPL23A group in mice were prominently decreased. To prove whether miR-1233/ACAT2 axis was responsible for circ_RPL23A action as in vitro, qRT-PCR and western blot were used for determining the levels of these three genes. As Fig. 7C exhibited, the high circ_RPL23A was detected in circ_RPL23A transfection group, implying that circ_RPL23A was successfully overexpressed in vivo. And miR-1233 level was reduced (Fig. 7D) while ACAT2, including mRNA and protein expression, was elevated (Fig. 7E-F) after circ_RPL23A promotion in vivo. Thus, circ_RPL23A was a CCRCC inhibitor relying on the miR-1233/ACAT2 axis in vivo, just like that in vitro.

\section{Discussion}

A recent study has reported the dysregulation of circRNAs in CCRCC and their potential clinical significance [22]. For instance, hsa_circ_001895 was an overexpressed circRNA [23] while circHIAT1 expression was down-regulated [24] in CCRCC. Wang et al. showed that hsa_circ_0001451 was lowly expressed in CCRCC cells and might be a useful target for the diagnosis and treatment of clinical patients [25]. In this chapter, circ_RPL23A was selected as a down-regulated gene through microarray analysis and 
its differential expression was also proved in CCRCC tissues and cells. As the result of close-loop structures, circRNAs are highly conserved and stable compared to the linear RNAs [26]. We found that circ_RPL23A was not easily affected by Actinomycin D and RNase treatment, testifying the identification of circ_RPL23A as a circRNA.

CircRNAs have the potential to promote or inhibit the progression of cancers. Up-regulation of hsa_circ_001895 acted as an oncogene to heighten cell proliferation, migration, invasion and suppress cell apoptosis of CCRCC [23]. Huang et al. declared that circ_ABCB10 enhanced cell proliferation and reduced cell apoptosis to facilitate CCRCC progression [27]. Differentially, circ-AKT3 overexpression was demonstrated to decrease migrated and invaded CCRCC cells [28]. CircRNA cRAPGEF5 [29] and has_circ_0072309 [30] also impeded tumor growth and metastasis in CCRCC. In conformity with these anti-cancer circRNAs, circ_RPL23A overexpression evoked cell cycle arrest, proliferation and metastasis inhibition, and apoptosis elevation. It was quite obvious that circ_RPL23A was a tumor repressor in CCRCC, for the first time to be discovered.

In the molecular level, the roles of circRNAs in cancer development are mainly attributable to the alternation of miRNAs. CircADAMTS13 inhibited cell proliferation in hepatocellular carcinoma by sponging miR-484 [31] and circRNA cTFRC could sponge miR-107 to accelerate the evolvement of bladder cancer [32]. For circ_RPL23A in CCRCC, miR-1233 was identified as a target for circ_RPL23A and circ_RPL23A played an anti-carcinoma role in CCRCC through the functional pathway of sponging miR1233.

MiRNAs have essential biological roles and clinical implication in CCRCC [33]. MiR-200c-3p was shown to restrain CCRCC cell migration and invasion by targeting SLC6A1 [34], and miR-122 acted as a tumor promoter of CCRCC via down-regulating FOXO3 [35]. Herein, we found that miR-1233 directly targeted ACAT2 to be a pro-cancer molecule of CCRCC. More interestingly, ACAT2 level also could be changed via circ_RPL23A-mediated miR-1233 inhibition. Thus, this circ_RPL23A/miR-1233/ACAT2 axis was established in regulating CCRCC progression, not only in vitro but also in vivo.

In conclusion, circ_RPL23A was proved to be a tumor inhibitor during CCRCC progression via the miR1233/ACAT2 axis in the current study. The circ_RPL23A/miR-1233/ACAT2 axis is firstly uncovered in CCRCC, which will afford the theoretical basis for molecular pathomechanism of CCRCC. All of the research purposes will eventually lead to clinical treatment, and circ_RPL23A may be a promising therapeutic biomarker for CCRCC.

\section{Abbreviations}

CCRCC

Clear cell renal cell carcinoma

ACAT2

acetyl-coenzyme A acetyltransferase 2

circ_RPL23A 


\section{Declarations}

\section{Acknowledgement}

None

\section{Funding}

None

\section{Availability of data and materials}

The data that support the findings of this study are available on request from the corresponding author.

\section{Authors' contributions}

Liang Cheng participated in the design of the work, methodology, data interpretation, and drafted the manuscript. Huifeng Cao and Jianbo Xu participated in the collection of data and analysis for the work, carried out the statistical analysis. Mo Xu and Wenjie He participated in the methodology, data interpretation. Wenjing Zhang and Longxin Dong participated in data interpretation and methodology. Dayin Chen interpreted the data and provided critical revisions. All authors read and approved the final manuscript.

\section{Ethics approval and consent to participate}

This study has got the authorization of the Ethical Committee of First Affiliated Hospital of Jiamusi University.

\section{Patient consent for publication}

Tissue collection was performed after the informed consent was signed and in line with the Declaration of Helsinki.

\section{Competing interests}

The authors declare that they have no competing interest.

\section{References}

1. Siegel RL, Miller KD, Jemal A. Cancer statistics, 2018. CA Cancer J Clin. 2018;68(1):7-30.

2. Hsieh JJ, Le V, Cao D, et al. Genomic classifications of renal cell carcinoma: a critical step towards the future application of personalized kidney cancer care with pan-omics precision. J Pathol. 2018;244(5):525-37. 
3. Li QK, Pavlovich CP, Zhang H, et al. Challenges and opportunities in the proteomic characterization of clear cell renal cell carcinoma (ccRCC): A critical step towards the personalized care of renal cancers. Semin Cancer Biol. 2019;55:8-15.

4. Siegel RL, Miller KD, Jemal A. Cancer Statistics, 2017. CA Cancer J Clin. 2017;67(1):7-30.

5. Jiang HM, Wei JH, Zhang ZL, et al. Does chromophobe renal cell carcinoma have better survival than clear cell renal cell carcinoma? A clinical-based cohort study and meta-analysis. Int Urol Nephrol. 2016;48(2):191-9.

6. Lee YT, Tan YJ, Oon CE. Molecular targeted therapy: Treating cancer with specificity. Eur J Pharmacol. 2018;834:188-96.

7. Barata P, Sood AK, Hong DS. RNA-targeted therapeutics in cancer clinical trials: Current status and future directions. Cancer Treat Rev. 2016;50:35-47.

8. Qu S, Yang X, Li X, et al. Circular RNA: A new star of noncoding RNAs. Cancer Lett. 2015;365(2):1418.

9. Zhao ZJ, Shen J. Circular RNA participates in the carcinogenesis and the malignant behavior of cancer. RNA Biol. 2017;14(5):514-21.

10. Chen B, Huang S. Circular RNA: An emerging non-coding RNA as a regulator and biomarker in cancer. Cancer Lett. 2018;418:41-50.

11. Ma C, Qin J, Zhang J, et al. Construction and analysis of circular RNA molecular regulatory networks in clear cell renal cell carcinoma. Mol Med Rep. 2020;21(1):141-50.

12. Yin $\mathrm{Y}$, Long $\mathrm{J}, \mathrm{He} \mathrm{Q}$, et al. Emerging roles of circRNA in formation and progression of cancer. $\mathrm{J}$ Cancer. 2019;10(21):5015-21.

13. Hammond SM. An overview of microRNAs. Adv Drug Deliv Rev. 2015;87:3-14.

14. Qiu Y, Pu C, Li Y, et al. Construction of a circRNA-miRNA-mRNA network based on competitive endogenous RNA reveals the function of circRNAs in osteosarcoma. Cancer Cell Int. 2020;20:48.

15. Sun X, Ge X, Xu Z, et al. Identification of circular RNA-microRNA-messenger RNA regulatory network in hepatocellular carcinoma by integrated analysis. J Gastroenterol Hepatol. 2020;35(1):157-64.

16. Zhou W, Wu G, Li J, et al. circRASSF2 Acts as ceRNA and Promotes Papillary Thyroid Carcinoma Progression through miR-1178/TLR4 Signaling Pathway. Mol Ther Nucleic Acids. 2020;19:1153-63.

17. Yadav S, Khandelwal M, Seth A, et al. Serum microRNA Expression Profiling: Potential Diagnostic Implications of a Panel of Serum microRNAs for Clear Cell Renal Cell Cancer. Urology. 2017;104:64-9.

18. Braga EA, Fridman MV, Loginov VI, et al. Molecular Mechanisms in Clear Cell Renal Cell Carcinoma: Role of miRNAs and Hypermethylated miRNA Genes in Crucial Oncogenic Pathways and Processes. Front Genet. 2019;10:320.

19. Zhao Z, Lu J, Han L, et al. Prognostic significance of two lipid metabolism enzymes, HADHA and ACAT2, in clear cell renal cell carcinoma. Tumour Biol. 2016;37(6):8121-30.

20. Yu J, Zhang L, Ren P, et al. Enterovirus 71 mediates cell cycle arrest in $S$ phase through non-structural protein 3D. Cell Cycle. 2015;14(3):425-36. 
21. Wang ZY, Zhong T, Wang Y, et al. Human Enterovirus 68 Interferes with the Host Cell Cycle to Facilitate Viral Production. Front Cell Infect Microbiol. 2017;7:29.

22. Franz A, Ralla B, Weickmann S, et al. Circular RNAs in Clear Cell Renal Cell Carcinoma: Their Microarray-Based Identification, Analytical Validation, and Potential Use in a Clinico-Genomic Model to Improve Prognostic Accuracy. Cancers (Basel). 2019;11(10).

23. Chen Z, Xiao K, Chen S, et al. Circular RNA hsa_circ_001895 serves as a sponge of microRNA-296-5p to promote clear cell renal cell carcinoma progression by regulating SOX12. Cancer Sci. 2020;111(2):713-26.

24. Wang $\mathrm{K}$, Sun $\mathrm{Y}$, Tao W, et al. Androgen receptor (AR) promotes clear cell renal cell carcinoma (ccRCC) migration and invasion via altering the circHIAT1/miR-195-5p/29a-3p/29c-3p/CDC42 signals. Cancer Lett. 2017;394:1-12.

25. Wang G, Xue W, Jian W, et al. The effect of Hsa_circ_0001451 in clear cell renal cell carcinoma cells and its relationship with clinicopathological features. J Cancer. 2018;9(18):3269-77.

26. Cai H, Li Y, Niringiyumukiza JD, et al. Circular RNA involvement in aging: An emerging player with great potential. Mech Ageing Dev. 2019;178:16-24.

27. Huang $Y$, Zhang $Y$, Jia $L$, et al. Circular RNA ABCB10 promotes tumor progression and correlates with pejorative prognosis in clear cell renal cell carcinoma. Int J Biol Markers. 2019;34(2):176-83.

28. Xue $D$, Wang $H$, Chen $Y$, et al. Circ-AKT3 inhibits clear cell renal cell carcinoma metastasis via altering miR-296-3p/E-cadherin signals. Mol Cancer. 2019;18(1):151.

29. Chen Q, Liu T, Bao Y, et al. CircRNA cRAPGEF5 inhibits the growth and metastasis of renal cell carcinoma via the miR-27a-3p/TXNIP pathway. Cancer Lett. 2020;469:68-77.

30. Chen T, Shao S, Li W, et al. The circular RNA hsa-circ-0072309 plays anti-tumour roles by sponging miR-100 through the deactivation of PI3K/AKT and mTOR pathways in the renal carcinoma cell lines. Artif Cells Nanomed Biotechnol. 2019;47(1):3638-48.

31. Qiu L, Huang Y, Li Z, et al. Circular RNA profiling identifies circADAMTS13 as a miR-484 sponge which suppresses cell proliferation in hepatocellular carcinoma. Mol Oncol. 2019;13(2):441-55.

32. Su H, Tao T, Yang Z, et al. Circular RNA cTFRC acts as the sponge of MicroRNA-107 to promote bladder carcinoma progression. Mol Cancer. 2019;18(1):27.

33. He YH, Chen C, Shi Z. The biological roles and clinical implications of microRNAs in clear cell renal cell carcinoma. J Cell Physiol. 2018;233(6):4458-65.

34. Maolakuerban N, Azhati B, Tusong H, et al. MiR-200c-3p inhibits cell migration and invasion of clear cell renal cell carcinoma via regulating SLC6A1. Cancer Biol Ther. 2018;19(4):282-91.

35. Nie W, Ni D, Ma X, et al. miR122 promotes proliferation and invasion of clear cell renal cell carcinoma by suppressing Forkhead box 03. Int J Oncol. 2019;54(2):559-71.

\section{Figures}


A

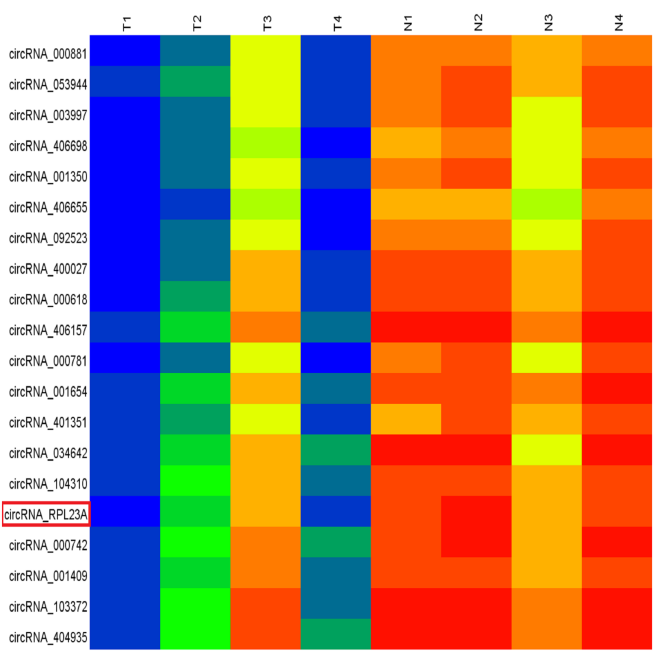

B

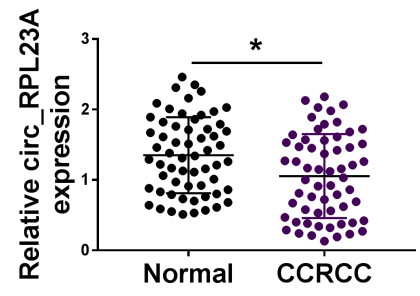

D

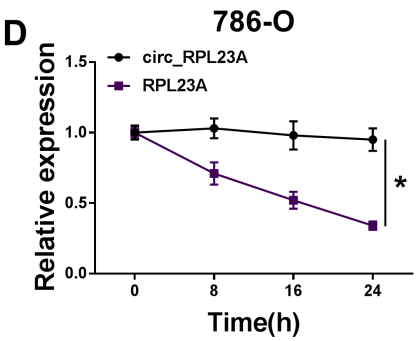

769-P
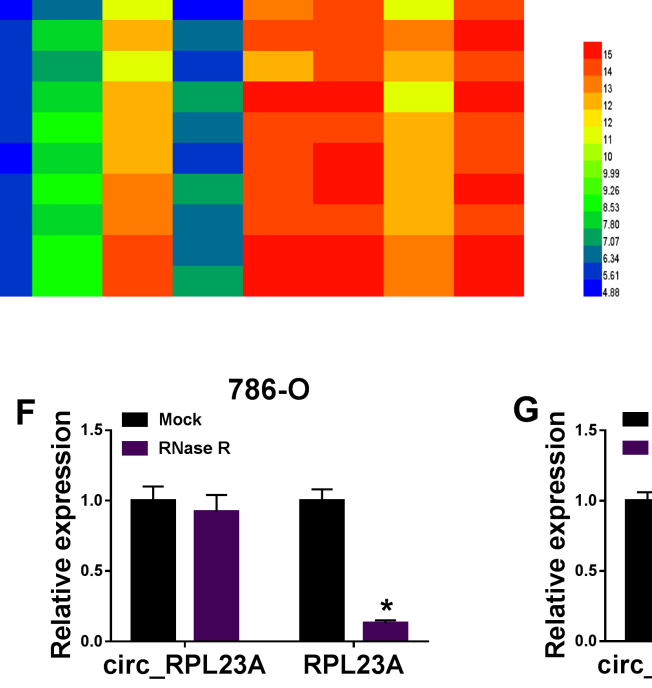

C
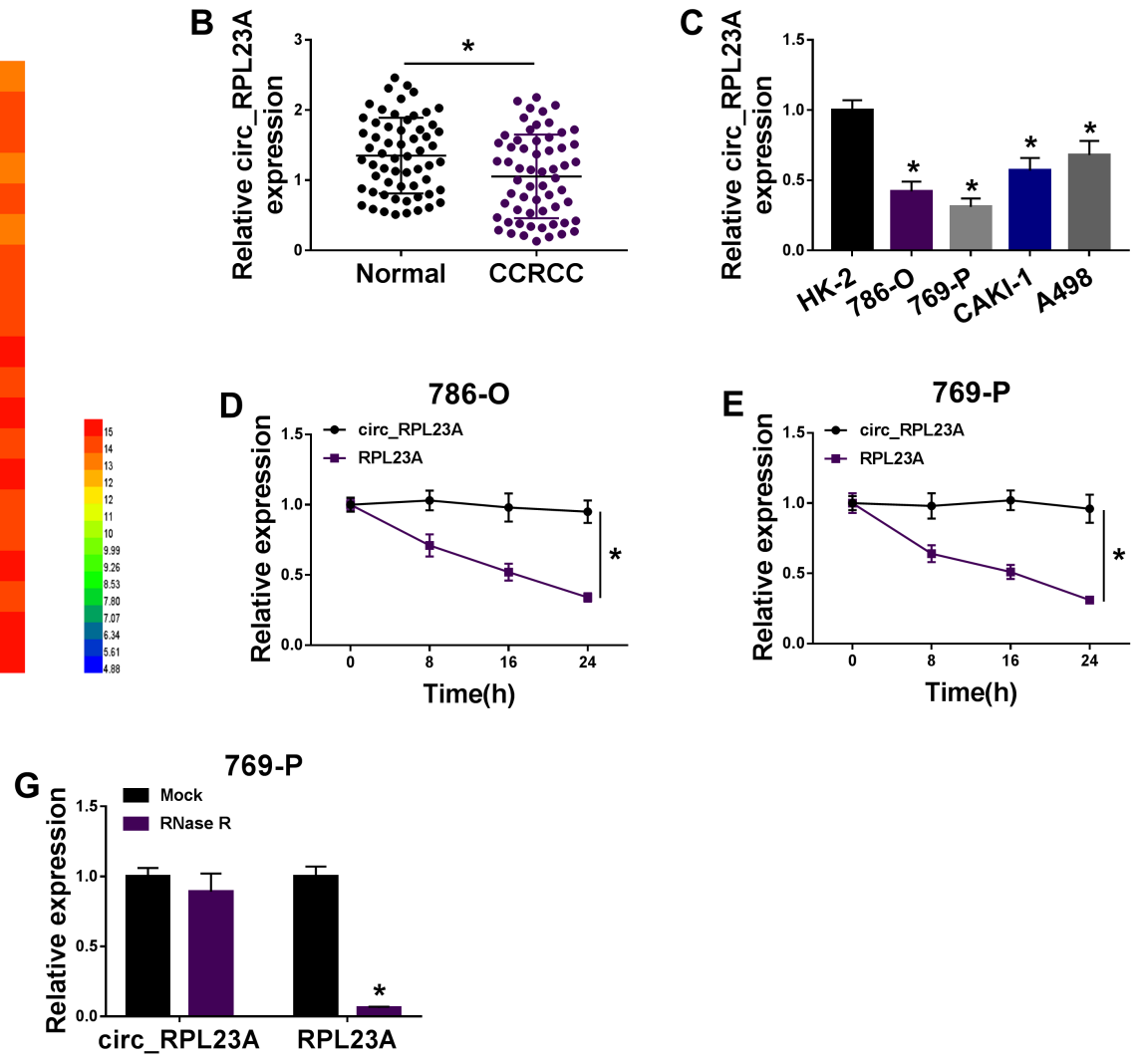

\section{Figure 1}

Circ_RPL23A down-regulation in CCRCC was confirmed. (A) Top dysregulated circular genes in CCRCC samples were presented in gene expression microarray. (B-C) The expression pattern of circ_RPL23A in CCRCC tissues (B) and cells (C) was examined applying with qRT-PCR. (D-G) After treatment of Actinomycin D (D-E) or RNase R (F-G), circ_RPL23A and RPL23A levels were analyzed via qRT-PCR. *P < 0.05 . 

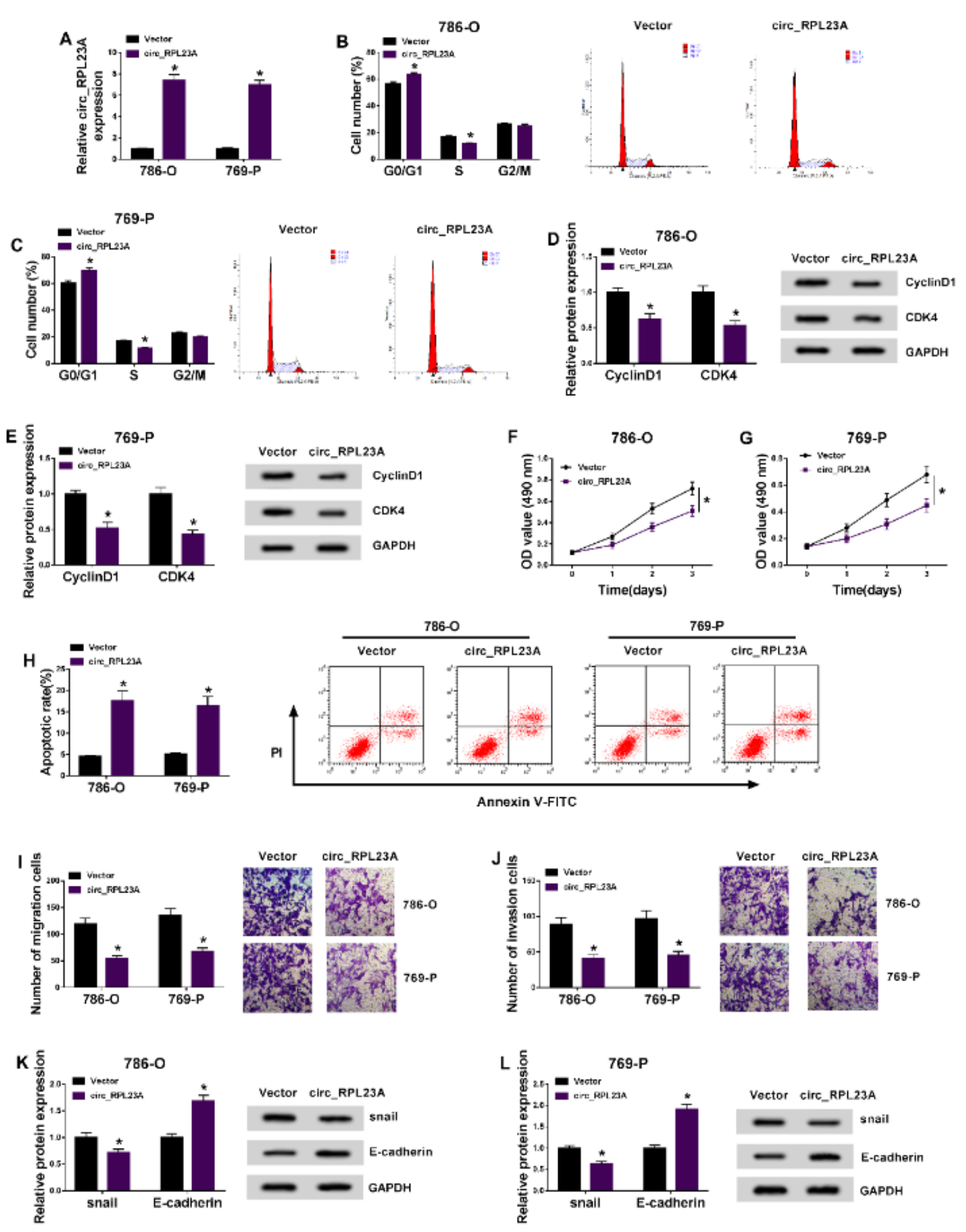

\section{Figure 2}

Overexpression of circ_RPL23A repressed tumor progression in CCRCC cells. (A) The transfection efficiency was assessed through qRT-PCR after circ_RPL23A or Vector transfection in 786-0 and 769-P cells. (B-C) Cell cycle of transfected cells was detected by PI-flow cytometry. (D-E) Western blot was administrated for determining cell cycle proteins CyclinD1 and CDK4 after transfection of circ_RPL23A or Vector. $(\mathrm{F}-\mathrm{H})$ Cell proliferation $(\mathrm{F}-\mathrm{G})$ and apoptosis $(\mathrm{H})$ were examined using MTT and Annexin V/PI-flow 
cytometry. (I-J) Transwell assay was used to measure the migrated and invaded cells. (K-L) The determination of snail and E-cadherin proteins was carried out via western blot. ${ }^{*} P<0.05$.
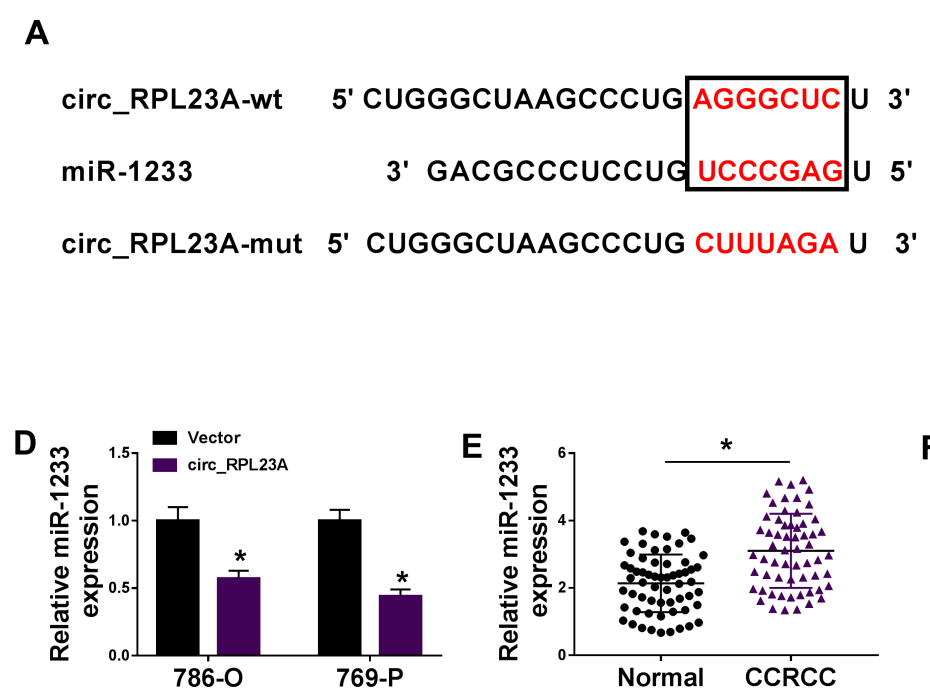


\section{Figure 3}

Circ_RPL23A was identified as a miR-1233 sponge in CCRCC. (A) Circinteractome was employed to present the binding sites between circ_RPL23A and miR-1233. (B-C) The luciferase activity was examined using dual-luciferase reporter system after co-transfection of circ_RPL23A-wt or circ_RPL23A-mut and miR-1233 or miR-NC. (D) The detection of miR-1233 was performed via qRT-PCR in 786-0 and 769-P cells transfected with circ_RPL23A or Vector. (E) The miR-1233 expression was assayed through qRT-PCR in CCRCC tissues and normal counterparts. (F) Spearman's correlation coefficient was used for the relation analysis between circ_RPL23A and miR-1233 in CCRCC tissues. (G) The expression analysis of miR-1233 in 786-0, 769-P and HK-2 cells was executed utilizing qRT-PCR. ${ }^{*} P<0.05$. 

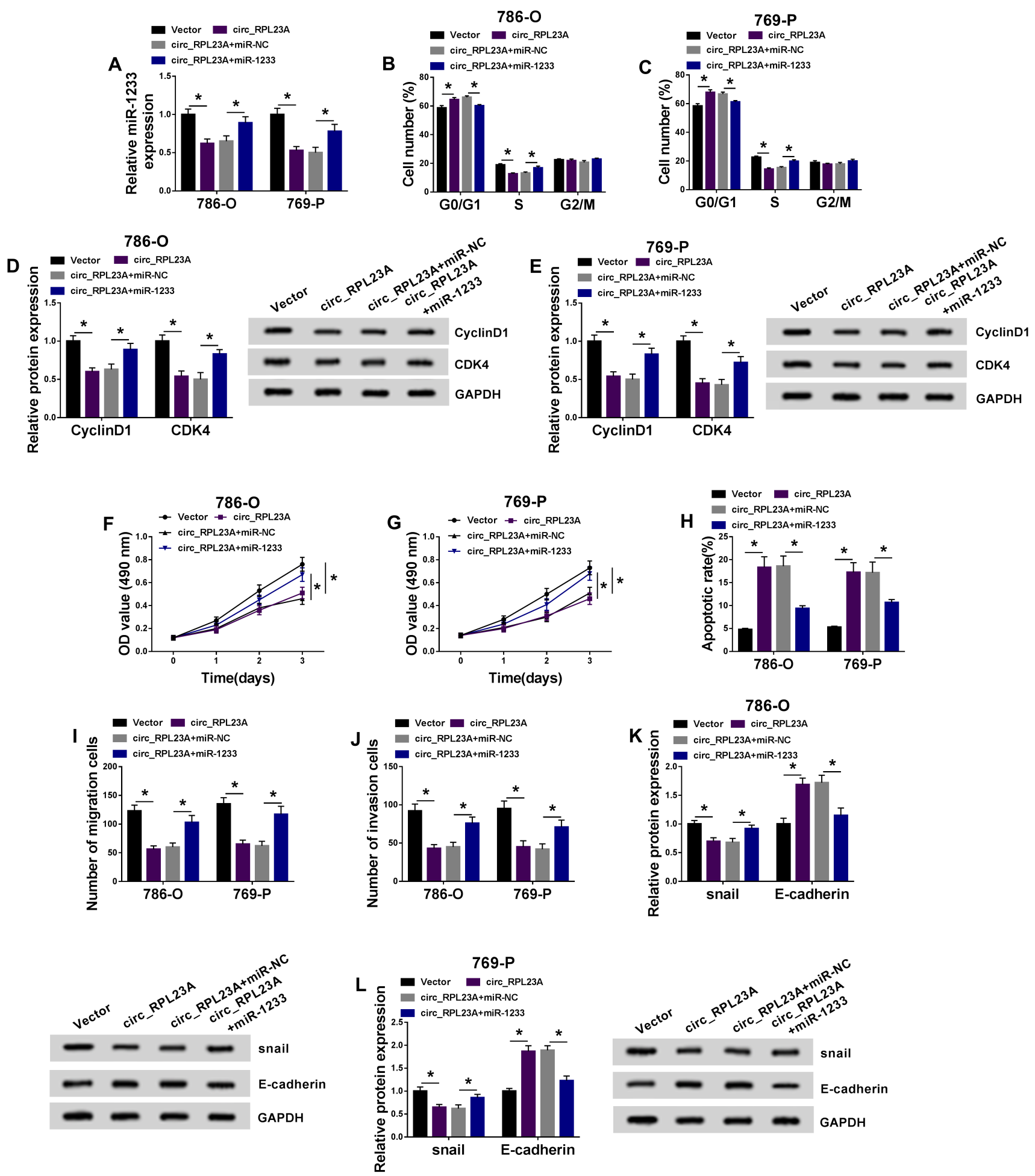

\section{Figure 4}

Circ_RPL23A/miR-1233 inhibited the development of CCRCC. (A) MiR-1233 was assayed via qRT-PCR in 786-0 and 769-P cells transfected with Vector, circ_RPL23A, circ_RPL23A+miR-NC or circ_RPL23A+miR1233. (B-E) PI-flow cytometry (B-C) and western blot (D-E) were applied for cell cycle analysis in above transfected cells. (F-G) The proliferation measurement was carried out via MTT. (H) Apoptotic rates of transfected cells were assayed using Annexin V/PI flow cytometry. (I-L) Cell metastasis was evaluated 
through transwell migration/invasion assays (I-J) and EMT protein detection by western blot (K-L). *P < 0.05 .

A

ACAT2 3' UTR

ACAT2-wt 5' TCAGATCAATCATTA AGGGCTC CA 3'

miR-1233 3' GACGCCCUCCUG UCCCGAG U 5'

ACAT2-mut 5' TCAGATCAATCATTA CUUUAGA CA 3'
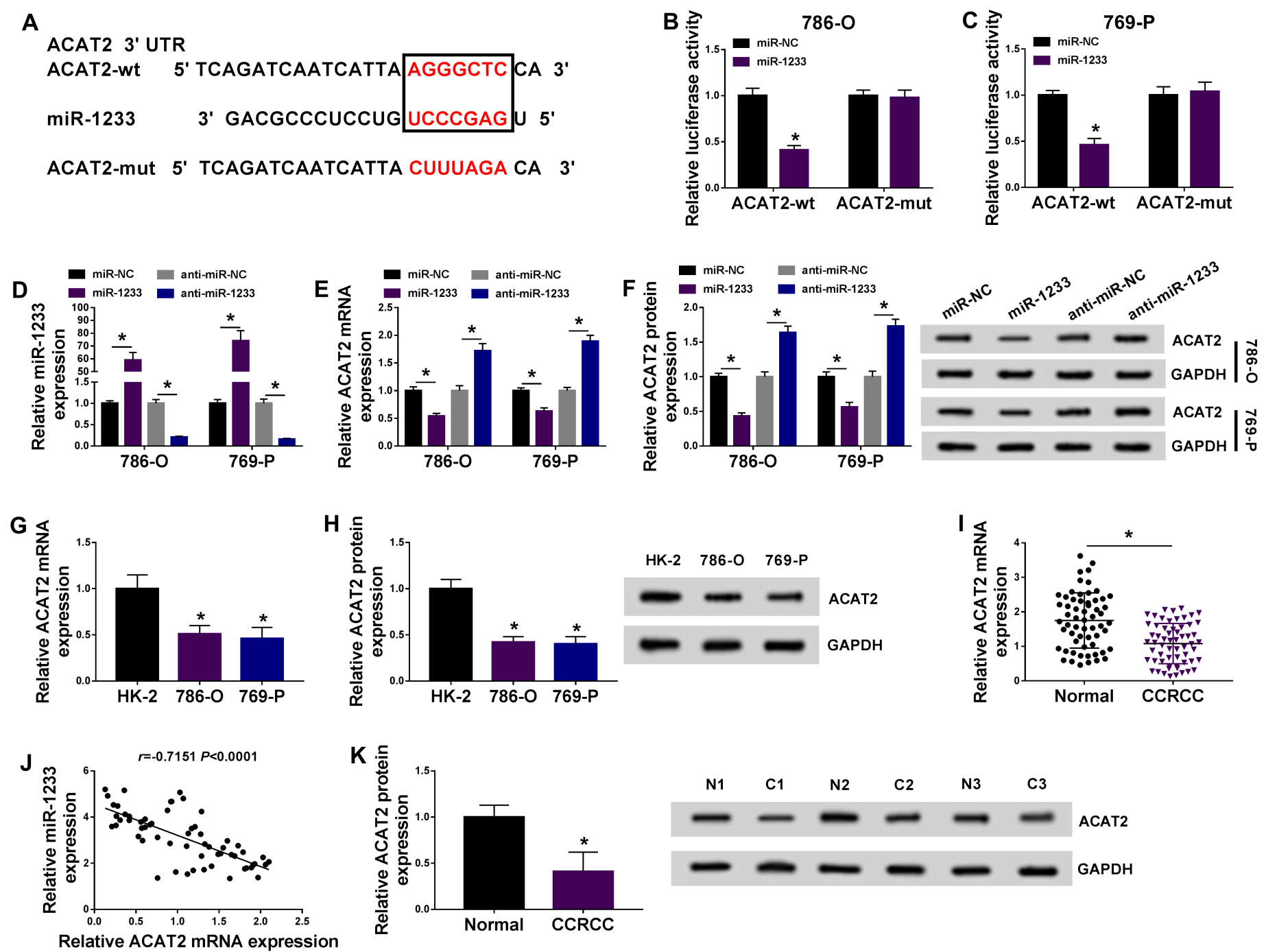

Relative ACAT2 mRNA expression
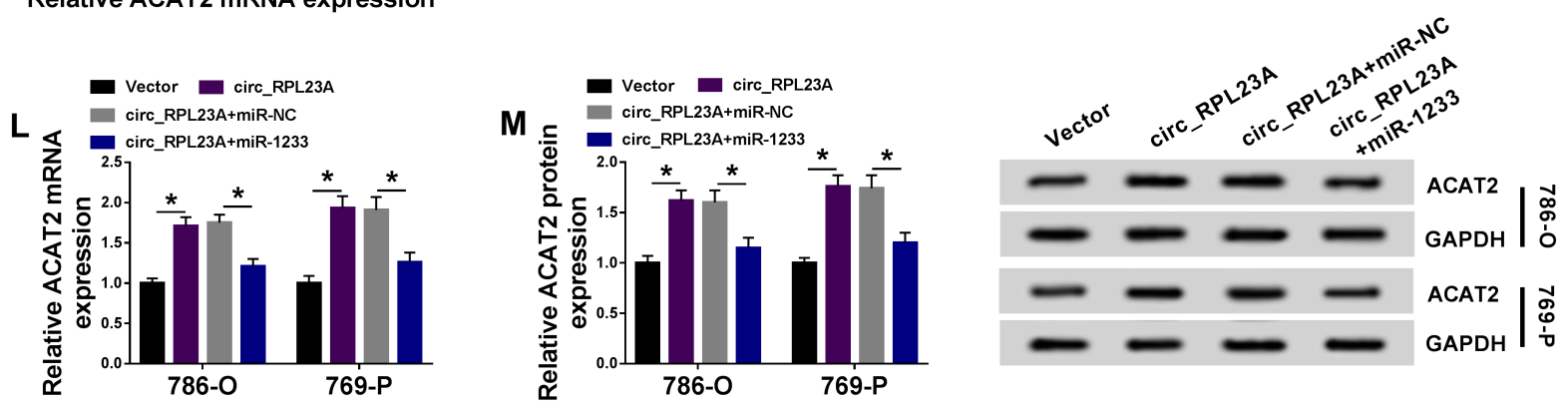

\section{Figure 5}

Circ_RPL23A enhanced ACAT2 via sponging miR-1233 in CCRCC. (A) The potential binding sites in the sequences of miR-1233 and 3'UTR of ACAT2 were predicted by miRDB. (B-C) Dual-luciferase reporter system was administrated for examining the relative luciferase intensity of ACAT2-wt or ACAT2-mut plasmid after miR-1233 or miR-NC transfection. (D) The qRT-PCR was applied to assess the transfection efficiency of miR-1233 and anti-miR-1233 using miR-NC and anti-miR-NC as respective control. (E-F) 
ACAT2 mRNA and protein levels were determined through qRT-PCR and western blot after miR-1233 overexpression and inhibition. (G-H) ACAT2 detection was implemented by qRT-PCR and western blot in 786-0, 769-P and normal HK-2 cells. (I) The mRNA expression of ACAT2 was analyzed via qRT-PCR in 60 CCRCC and normal tissues. $(\mathrm{J})$ The analysis of linear association between miR-1233 and ACAT2 was conducted depending on Spearman's correlation coefficient. (K) Western blot was executed for ACAT2 protein level analysis in CCRCC tissues. (L-M) The effects of circ_RPL23A/miR-1233 on ACAT2 mRNA and protein levels were measured via qRT-PCR and western blot. ${ }^{*} \mathrm{P}<0.05$.
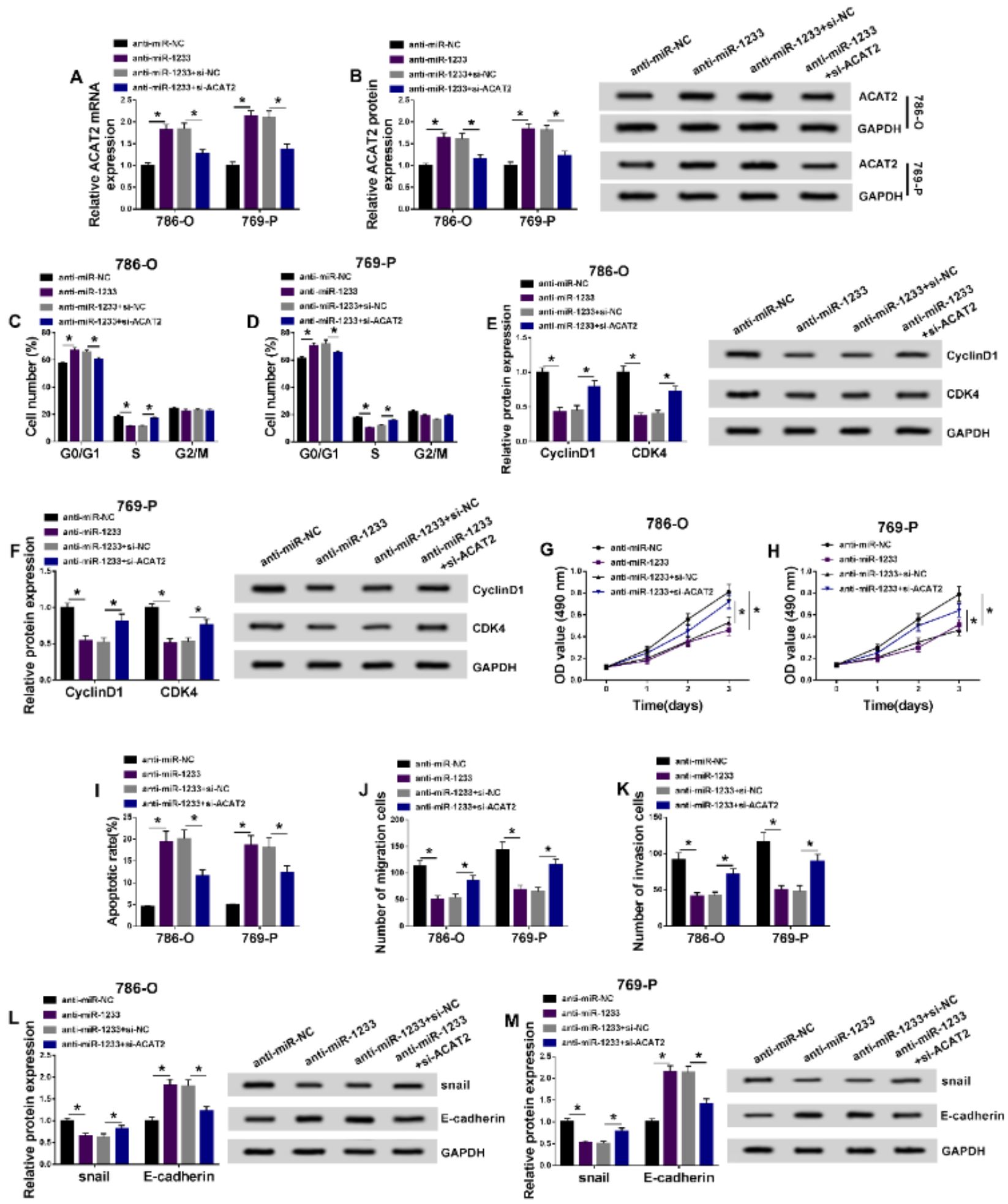


\section{Figure 6}

MiR-1233 played a tumor-promoting role in via targeting ACAT2 in CCRCC. (A-B) ACAT2 mRNA and protein levels of anti-miR-NC, anti-miR-1233, anti-miR-1233+si-NC or anti-miR-1233+si-ACAT2 group in 786-0 and 769-P cells were assayed using western blot. (C-F) The examination of cell cycle was implemented using PI-flow cytometry (C-D) and protein analysis (E-F). (G-H) MTT was used for proliferation analysis of transfected cells. (I) The influence of above transfection on cell apoptosis was analyzed by Annexin V/PI flow cytometry. (J-M) Transwell migration/invasion assays (J-K) and EMT protein analysis $(\mathrm{L}-\mathrm{M})$ were utilized for assessing the metastatic ability of transfected cells in above. ${ }^{*}<$ 0.05 .
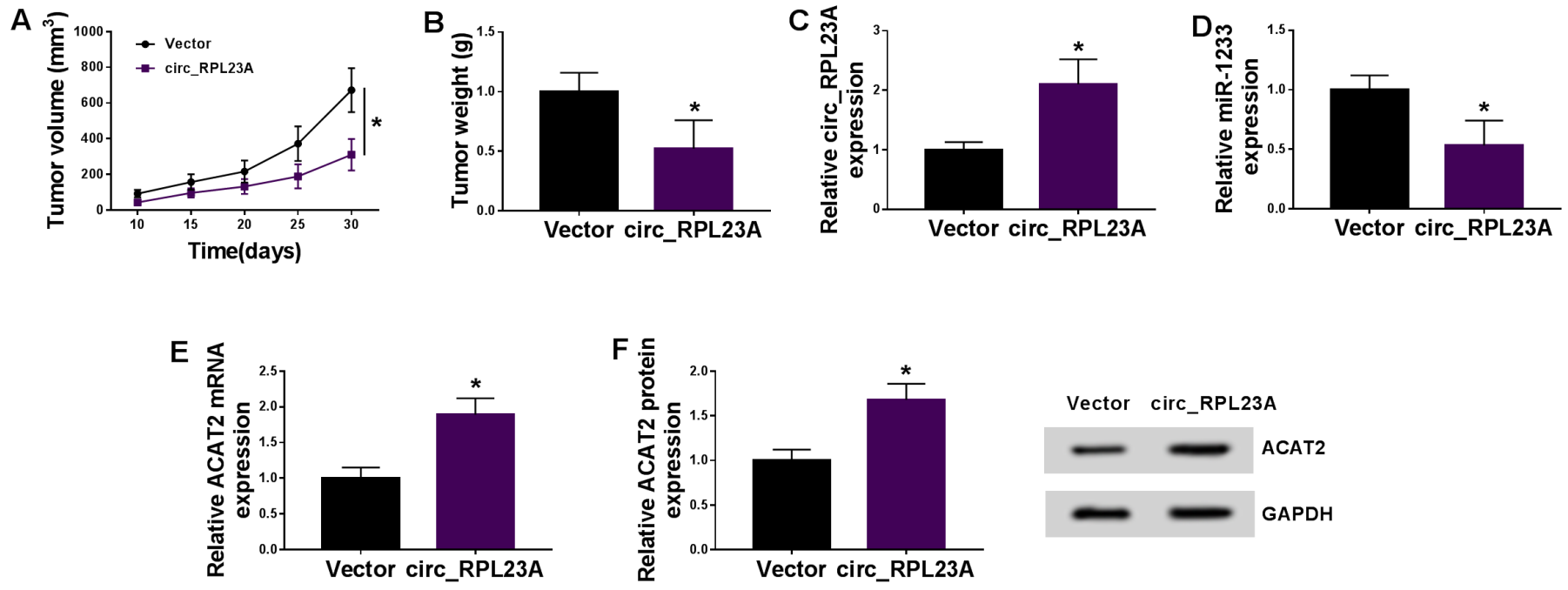

\section{Figure 7}

Circ_RPL23A worked as a tumor inhibitor of CCRCC in vivo by ACAT2 up-regulation as a miR-1233 sponge. (A-B) A vernier caliper and an electronic scale were respectively used to measure tumor volume (A) and weight (B). (C-E) The qRT-PCR was exploited to examine the expression of circ_RPL23A (C), miR1233 (D) and ACAT2 mRNA (E). (F) ACAT2 protein level was analyzed employing western blot. * $\mathrm{P}<0.05$. 